\title{
Modified Protocol for Yeast Identification Using Matrix- Assisted Laser Desorption Ionization-Time of Flight Mass Spectrometry
}

\author{
Janet W. S. Cheng, Yit Er Tang, Roland Jureen, Raymond T.P. Lin, Jeanette W.P. Teo* \\ Department of Laboratory Medicine, Microbiology Unit, National University Hospital, Singapore, Republic of Singapore \\ *Corresponding author: Jeanette_Teo@nuhs.edu.sg
}

Received August 19, 2013; Revised August 26, 2013; Accepted October 09, 2013

\begin{abstract}
Advances in Matrix-assisted Laser Desorption Ionization-time of Flight Mass Spectrometry (MALDIToF MS) identification methods have greatly facilitated the rapid identification of yeasts. We validate the use of a shorter on-plate extraction method as well as a lower score cutoff value on the Bruker Biotyper. Overall, when the on-plate extraction method was used together with a score cutoff of $>1.7$, a total of 151 of 187 (81\%) Candida isolates were correctly identified. At species level, almost $100 \%$ of C. krusei and C. albicans isolates were correctly identified. We find the revised protocol to be effective for routine identification of yeasts.
\end{abstract}

Keywords: protein extraction, Candida, identification scores

Cite This Article: Janet W. S. Cheng, Yit Er Tang, Roland Jureen, Raymond T.P. Lin, and Jeanette W.P. Teo, "Modified Protocol for Yeast Identification using Matrix-Assisted Laser Desorption Ionization-Time of Flight Mass Spectrometry." American Journal of Microbiological Research 1, no. 4 (2013): 71-73. doi: 10.12691/ajmr$1-4-2$.

\section{Introduction}

Matrix-assisted laser desorption ionization-time of flight mass spectrometry (MALDI-ToF MS) has become the workhorse in clinical microbiology laboratories for the rapid identification of microorganisms [1]. The instrument identifies microbes by analyzing proteins released from the cells against its reference database of profiles. Identification of Gram-negative bacteria is achieved fairly effortlessly through the direct method of analysis where colonies are smeared as a thin layer of cells onto the target plate followed by overlaying matrix onto the cell layer whereas the identification of yeast and Gram-positive bacteria is more onerous. Yeast cells require a harsher preprocessing step for the release of cellular proteins [2]. The standard tube-based method of protein extraction, regarded as the 'gold-standard' for MALDI-ToF MS extractions, employs formic acid-acetonitrile treatment and multiple centrifugation steps in an Eppendorf tube before finally transferring processed sample onto the target plate. This method achieves close to $100 \%$ correct identification of yeasts $[3,4]$ but takes about 25 minutes or 6-fold more time than the direct method. The standard method of extraction is too tedious, demanding excessive hands-on time for a clinical laboratory. A shorter (15 minutes) and uncomplicated alternative to the standard extraction method described as on-plate extraction has been found to be comparable to the standard method for providing correct identifications [5,6,7].

Several validation studies have indicated that it is acceptable to revise the manufacturer-recommended interpretive criteria for yeasts. The Bruker MALDI Biotyper system, for example, recommends an organism score of greater than 2.0 to report a species identification. Scores of between 1.7 and 1.99 provide confidence to the genus level, and scores below 1.7 are not reliable. This criterion has been found to be overly conservative for certain microbes groups including the yeasts $[4,8]$. Studies have found that scores $>1.7$ for Candida spp. were accurate for diagnosis to the species level $[4,6,9,10]$.

In this study, we wanted to validate for yeasts: (i) the on-plate method of extraction and (ii) the lowering of the interpretative cutoff for species level to $>1.7$, instead of the manufacturer's (Bruker Daltonik) recommended score of $>2$.

\section{Materials and Methods}

\subsection{Candida Isolates and Phenotypic Identification}

The Candida isolates were obtained from patients with Candida infections and cultured onto Sabouraud's dextrose agar (Difco, MI, USA). A total of 187 clinical isolates from 5 different species were studied (Table 1). Candida spp. were identified phenotypically by inoculating all isolates onto BBL CHROMagar Candida (Becton Dickinson, Heidelberg, Germany). Colony growth and color development was noted on CHROMagar Candida. Green and blue colonies were identified as $C$. albicans andC. tropicalis, respectively. Colonies producing colors apart from green and blue were 
identified on the automated system for microorganism identification using VITEK 2 YST card (bioMérieux, Marcy l'Etoile, France). Inconclusive results were resolved by identification with API 20 C AUX (bioMérieux).

Table 1. Breakdown of result scores obtained for yeast isolates subjected to three different extraction methods (direct colony, on-plate extraction and standard tube extraction method)

\begin{tabular}{|c|c|c|c|c|}
\hline & \multicolumn{4}{|c|}{ No. of isolates producing a score of (\%) } \\
\hline & $\geq 2$ & $1.7-1.99$ & $<1.7$ & Invalid identifications \\
\hline \multicolumn{5}{|c|}{ Candida albicans $(n=49)$} \\
\hline Direct & $1(2)$ & $21(42.9)$ & $5(10.2)$ & 22 (44.9) \\
\hline On-plate & $29(59.2)$ & $19(38.8)$ & 0 & $1(2)$ \\
\hline Standard & $40(81.6)$ & $9(18.4)$ & 0 & 0 \\
\hline \multicolumn{5}{|c|}{ Candida tropicalis $(n=50)$} \\
\hline Direct & $3(6)$ & $1(2)$ & $10(20)$ & $36(72)$ \\
\hline On-plate & $33(66)$ & $11(22)$ & $3(6)$ & $3(6)$ \\
\hline Standard & $35(70)$ & $9(18)$ & $5(10)$ & $1(2)$ \\
\hline \multicolumn{5}{|c|}{ Candida glabrata $(n=50)$} \\
\hline Direct & 0 & $1(2)$ & $26(52)$ & $23(46)$ \\
\hline On-plate & 0 & $28(56)$ & $18(36)$ & $4(8)$ \\
\hline Standard & $44(88)$ & $1(2)$ & $3(6)$ & $2(4)$ \\
\hline \multicolumn{5}{|c|}{ Candida parapsilosis $(n=30)$} \\
\hline Direct & 0 & $4(13.3)$ & $9(30)$ & $17(56.7)$ \\
\hline On-plate & $5(16.7)$ & $18(60)$ & 4 (13.3) & $3(10)$ \\
\hline Standard & $16(53.3)$ & 7 (23.3) & $4(13.3)$ & $3(10)$ \\
\hline \multicolumn{5}{|c|}{ Candida krusei $(n=8)$} \\
\hline Direct & 0 & 0 & $3(37.5)$ & $5(62.5)$ \\
\hline On-plate & $2(25)$ & $6(75)$ & 0 & 0 \\
\hline Standard & $8(100)$ & 0 & 0 & 0 \\
\hline
\end{tabular}

\subsection{MALDI-ToF Extraction and Identification}

an isolate had a correct identification when results showed that the first and second species identification was the same, with a score of at least $\geq 1.7$. The following were considered as invalid when (i) the first and second identification result were different (ii) the misidentification was obvious, for example, a bacterial identification is suggested (iii) no spectral peaks were generated. American type culture collection (ATCC) isolates of $C$. albicans 14053 and C. glabrata 15126 were used as positive controls for the analysis. These isolates were correctly identified to species level (score values $>1.8$ ) but were not included in the final analysis. Docs/Separations_MassSpectrometry/InstructionForUse/I FU_268711_267615_226413_MALDI_Biotarget_48_Rev 1.pdf. Direct colony method involved smearing a small amount of the yeast colony onto the MSP 96 target plate (Bruker Daltonik) using a $1 \mu \mathrm{L}$ disposable inoculation loop. The sample was overlaid with $1 \mu \mathrm{L}$ of HCCA matrix (a-cyano-4-hydroxycinnamic acid [Vitek MS-CHCA 41107] in $50 \%$ acetonile and $1.5 \%$ trifluoroacetic acid) and dried at room temperature. The on-plate extraction method involved the smearing of a thin layer of isolate onto the target plate followed by the addition of $1 \mu \mathrm{l}$ of $70 \%$ formic acid which was allowed to dry, $1 \mu \mathrm{l}$ of acetonitrile was overlaid and permitted to dry before applying $1 \mu \mathrm{l}$ of HCCA matrix. In the standard tubeextraction protocol, colonies were thoroughly resuspended in $300 \mu \mathrm{l}$ of distilled water and $900 \mu \mathrm{l}$ of absolute ethanol was added. The cell suspension was vortexed and centrifuged at 13, $000 \mathrm{rpm}$ for $2 \mathrm{~min}$. The supernatant was removed and $50 \mu \mathrm{l}$ of formic acid was added and the mixture was vortexed. Acetonitrile $(50 \mu \mathrm{l})$ was added and vortexed vigorously and the tube was centrifuged at 13 , $000 \mathrm{rpm}$ for $2 \mathrm{~min}$. One $\mu \mathrm{l}$ of the supernatant was spotted onto the target plate and allowed to dry before overlying with $1 \mu$ l of HCCA matrix.

The samples prepared by each method were applied to a MicroFlex LT mass spectrometer (Bruker Daltonik), and the results were analyzed by MALDI Biotyper 3.0 software (Bruker Daltonik) in automatic mode at manufacturer's setting. If the score identification was low $(<1.7)$ then the sample analysis was repeated. In our study,

\section{Statistical Analysis}

Comparisons of extraction methods for species level identifications were performed using McNemar's test for paired samples. $P$ values less than 0.05 were considered statistically significant.

\section{Results and Discussion}

In this study, we validate the use of a shorter on-plate extraction method as well as a lower score cutoff value on the Bruker MALDI-ToF Biotyper. The identification rates and breakdown of score categories using the 3 (see above) different extraction methods are shown in Table 1 . In the score category of $\geq 2$, the standard extractions produced the greatest number of correct identifications. This was $100 \%$ for C. krusei followed by $88 \%$ for C. glabrata and $81 \%$ for C. albicans. Compared to standard extractions, the on-plate method yielded fewer correct identifications whilst the direct method generated the poorest results for this score category, yielding no identifications for $C$. krusei, C. parapsilosis and C. glabrata and $<6 \%$ successful identifications for the other species (Table 1).

For the score category of 1.7 to 1.99 , the majority of identifications made were produced via the on-plate method. For C. krusei, C. parapsilosis, and C. glabrata, 
$75 \%, 60 \%$ and $56 \%$, respectively, of isolates were correctly identified using the on-plate procedure. The direct colony method identified less than $13.3 \%$ of isolates in four different species although C. albicans was an exception, having $42.9 \%$ of reliable identifications (Table 1).

Low scoring results $(<1.7)$ and invalid identifications were typically generated by the direct colony method. Misidentifications ranging from between $44.9 \%$ to $72 \%$ for the different species were produced whereas the onplate and standard methods had less than $10 \%$ misidentifications in this category (Table 1).

For the Bruker MALDI Biotyper system, lowering the species-secure score cutoff to $>1.7$ instead of the manufacturer's recommendation of $\geq 2.0$ is now deemed acceptable $[4,6,9,10]$. In our study, when the cutoff for identification to species level was modified to $>1.7$, we find that the level of correct identifications across the different species rises from $66 \%$ up to $100 \%$ for the onplate extraction method (Figure 1). Statistically, the onplate method had a similar rate of identification compared to the standard extraction method $(P=>0.05)$ (Figure 1 ) with the exception of $C$. glabrata. For this species, the identification using on-plate method of extraction was inferior to the standard method of extraction $(P=0.0001)$. The direct colony method was significantly inferior $(\mathrm{P}<$ 0.0001 ) in providing accurate species identification for all Candida analyzed (Figure 1).

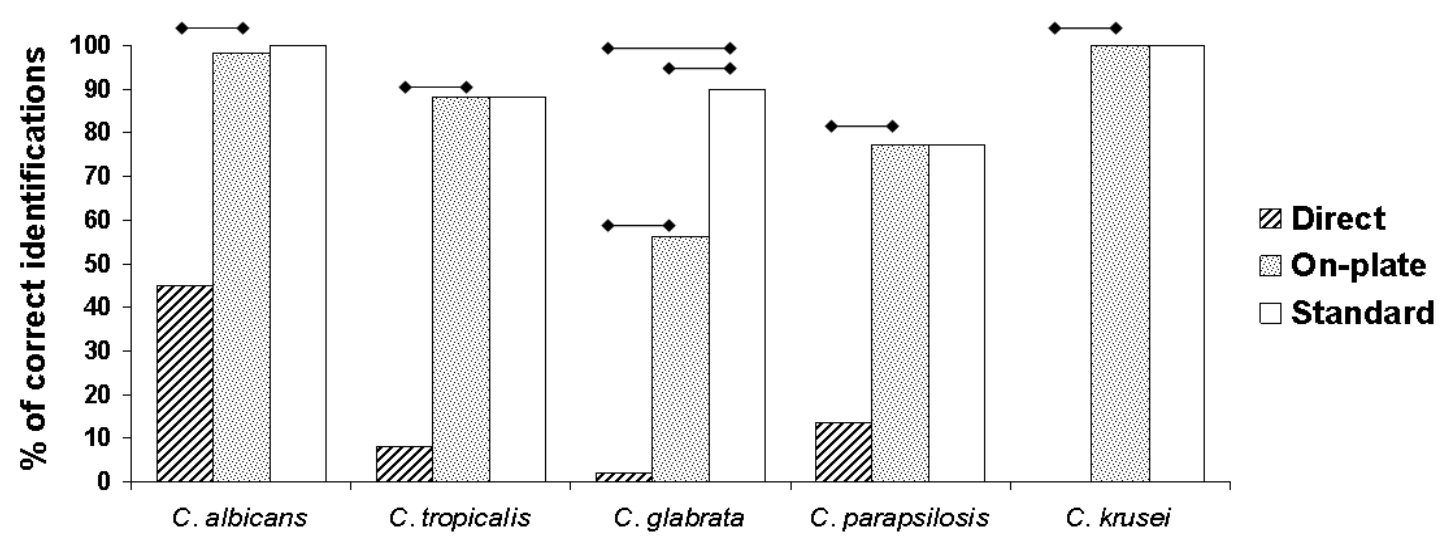

Figure 1. Bar plots showing the percentage of correct yeast identifications using three different extraction methods (direct colony, on-plate and standard-tube extraction methods) and a revised score cutoff of $>1.7$ for species-level identification. $\bullet$ Above the bars indicate statistically significant pairs

\section{Concluding Remarks}

Prompt and reliable species identification of Candida is critical to the treatment of candidiasis. C. glabrata has less susceptibility to fluconazole andC. krusei is intrinsically resistant to this drug hence differential therapeutic regimens may be required for the various species [11]. Our findings correlate well to investigations performed by other groups where the on-plate method of extraction coupled with a modified species score of $>1.7$, is nontedious, fast and reliable approach $[4,6,9,10]$. It is therefore highly suited for the workflow of the clinical microbiology laboratory for the identification of yeast isolates.

\section{References}

[1] Wieser, A., Schneider, L., Jung, J. and Schubert, S, "MALDI-TOF MS in microbiological diagnostics-identification of microorganisms and beyond (mini review)," Applied Microbiology and Biotechnology, 93 (3). 965-974. Feb. 2012.

[2] Buchan, B.W. and Ledeboer, N.A, "Advances in identification of clinical yeast isolates by use of matrix-assisted laser desorption ionization-time of flight mass spectrometry," Journal of Clinical Microbiology, 51 (5). 1359-1366. May. 2013.

[3] Bader, O., Weig, M., Taverne-Ghadwal, L., Lugert, R., Gross, U. and Kuhns M, "Improved clinical laboratory identification of human pathogenic yeasts by matrix-assisted laser desorption ionization time-of-flight mass spectrometry," Clinical Microbiology and Infection, 17 (9). 1359-1365. Sep. 2011.

[4] Dhiman, N., Hall, L., Wohlfiel, S.L., Buckwalter, S.P. and Wengenack N.L, "Performance and cost analysis of matrixassisted laser desorption ionization-time of flight mass spectrometry for routine identification of yeast," Journal of Clinical Microbiology, 49 (4). 1614-1616. Apr. 2011.

[5] Theel, E.S., Schmitt, B.H., Hall, L., Cunningham, S.A., Walchak R.C., Patel R. and Wengenack, N.L, "Formic acid-based direct, on-plate testing of yeast and Corynebacterium species by Bruker Biotyper matrix-assisted laser desorption ionization-time of flight mass spectrometry," Journal of Clinical Microbiology, 50 (9). 3093-3095. Jul. 2012.

[6] Van Herendael, B.H., Bruynseels, P., Bensaid, M., Boekhout, T., De Baere T, Surmont, I. and Mertens, A.H, "Validation of a modified algorithm for the identification of yeast isolates using matrix-assisted laser desorption/ionisation time-of-flight mass spectrometry (MALDI-TOF MS)," European Journal of Clinical Microbiology and Infectious Diseases, 31 (5). 841-848. May. 2012.

[7] Iriart, X., Lavergne, R.A., Fillaux, J., Valentin, A., Magnaval, J.F., Berry, A. and Cassaing, S, "Routine identification of medical fungi by the new Vitek MS matrix-assisted laser desorption ionization-time of flight system with a new time-effective strategy,” Journal of Clinical Microbiology, 50 (6). 2107-2110. Jun. 2012.

[8] Stevenson, L.G., Drake, S.K., Shea, Y.R., Zelazny, A.M. and Murray, P.R, "Evaluation of matrix-assisted laser desorption ionization-time of flight mass spectrometry for identification of clinically important yeast species," Journal of Clinical Microbiology, 48 (10). 3482-3486. Oct. 2010.

[9] Pinto, A., Halliday, C., Zahra, M., van Hal, S., Olma, T., Maszewska, K., Iredell, J.R., Meyer, W. and Chen, S.C, "Matrixassisted laser desorption ionization-time of flight mass spectrometry identification of yeasts is contingent on robust reference spectra," PLoS One, 6 (10).e25712. Oct. 2011.

[10] Cassagne, C., Cella, A.L., Suchon, P., Normand, A.C., Ranque, S. and Piarroux, R, "Evaluation of four retreatment procedures for MALDI-TOF MS yeast identification in the routine clinical laboratory,” Medical Mycology, 51 (4). 371-377. May. 2013.

[11] Bodey, G.P. 1992. Antifungal agents. In Candidiasis: pathogenesis, diagnosis and treatment, pp 371-406. Raven Press, New York, N.Y, 2nd ed. 